\title{
The dimensions of tonal experience: A nonmetric multidimensional scaling approach
}

\author{
BRUCE A. SCHNEIDER and RANDALL J. BISSETT \\ University of Toronto, Toronto, Ontario M5S 7A5, Canada
}

\begin{abstract}
Subjects were required in each trial to directly compare two pairs of tones and indicate which pair of tones had the greater subjective difference or dissimilarity. Eleven tones differing in both intensity and frequency were employed. Subjects made binary comparisons among the 55 tone pairs which can be formed from the set of 11 tones. These paired comparisons of tonal intervals were used to determine a two-dimensional Euclidean representation for tonal experience. Loudness and pitch appeared as orthogonal dimensions in this representation. However, a 45deg rotation of loudness and pitch axes produced axes which could be identified as volume and density. This relationship suggested that volume and density were simple functions of pitch and loudness. Volume and density predictions based on this two-dimensional representation were shown to provide a good account of the data from three experiments on volume and density.
\end{abstract}

While pure tones can be specified physically in terms of their frequency and intensity, it has been suggested (see Stevens, 1934) that it might take as many as four psychological dimensions (pitch, loudness, volume, and density) to represent them experientially. This creates a curious situation in which there appear to be more psychological dimensions than there are physical attributes. Normally this does not create a problem, since these psychological attributes are usually studied independently of one another. However, the dimensionality of tonal experience must be considered in any kind of perceptual judgment that may involve more than one of these psychological dimensions. This is clearly the case when one considers judgments of tonal dissimilarity.

If two pure tones differing in both frequency and intensity are presented sequentially, a subjective impression of tonal dissimilarity or difference results. In theory, the magnitude of this dissimilarity should be some joint function of the difference between these two tones along one or more of the relevant psychological dimensions. A dimensional analysis of this sort suggests a geometrical representation for tonal experience with loudness, pitch, volume, and density as dimensions in a tonal space. The dimensionality of this space could be as high as four if all four dimensions turned out to be mutually orthogonal. A pure tone would be represented as a point in this four-dimensional space, with its location determined by its loudness, pitch, volume, and den-

This research was supported by a grant from the Natural Sciences and Engineering Research Council of Canada. Reprints may be obtained from Bruce Schneider, Department of Psychology, Erindale College, University of Toronto, Mississauga, Ontario LSL 1C6, Canada. sity values. We would also expect that the distance between any two points in this space would reflect tonal dissimilarity.

However, there are suggestions in the literature that these four dimensions are not linearly independent. Stevens, Guirao, and Slawson (1965) demonstrated a systematic relationship between magnitude estimates of the volumes, densities, and loudness of narrow-band noises. They found that the loudness estimates were roughly the product of the density and volume estimates. If this systematic relationship were to characterize any arbitrary selection of pure tones, it would indicate, at the very least, that volume and density are not linearly independent of loudness, and suggest that the dimensionality of tonal experience was less than four. In fact, two previous studies have indicated that only two dimensions may be necessary to represent tonal dissimilarity. Carvellas and Schneider (1972) found that a two-dimensional auditory space with loudness and pitch as dimensions was able to account for magnitude estimates of the dissimilarity of pure tones varying in frequency and intensity. Chipman and Carey (1975) also found that a twodimensional auditory space could account for magnitude estimates of the dissimilarity of narrow-band noises varying in intensity and center frequency. However, their data clearly reject pitch as a dimension of their two-dimensional space and suggest that volume and density, or one of these dimensions in combination with loudness, serve as dimensions in their space. Thus, while these two studies agree on the dimensionality of auditory space, they disagree on the identity of the dimensions.

Hence, the existing literature is not clear as to how the dimensions of auditory experience enter into 
judgments of dissimilarity or perceived difference. Furthermore, while two studies suggest that a twodimensional representation is adequate to account for judgments of tonal dissimilarity, the identity of the two dimensions remains unresolved. Since the two studies employed different stimuli (pure tones vs. narrow-band noises), it is possible that this difference might account for the fact that pitch appeared as a dimension of dissimilarity judgments for pure tones but not for narrow-band noises. Another possibility that must be considered is that individual subjects differ with respect to the auditory dimensions that contribute to judgments of dissimilarity. If sounds differ along four psychological dimensions, it may be the case that the contribution of each of these dimensions to tonal dissimilarity differs from one subject to the next. Thus, averaging across subjects (as was done in Chipman \& Carey, 1975) might result in a space that does not adequately represent any of these dimensions.

A final possibility is that the two-dimensional configurations obtained in one or both of these studies are distorted by the inability of subjects to accurately assign numbers to represent the magnitude of the dissimilarity between two sounds that vary along more than one psychological attribute. To obtain a perfect spatial representation of dissimilarity judgments, nonmetric scaling techniques require that these judgments be monotonically related to distances in a spatial representation. If numerical estimates of dissimilarities are highly variable or unstable, the function relating judgments to spatial distances will not be completely monotonic. In that case, the value of stress (an index of the degree of nonmonotonicity) will be high and the obtained configuration will not accurately reflect the true configuration (Young, 1970). The high stress values found in both Carvellas and Schneider (1972) and Chipman and Carey (1975) indicate that this may, indeed, have been the case. Consequently, it would seem reasonable to employ a scaling technique that would not require that subjects generate numbers to represent dissimilarities.

In the present study, numerical biases are avoided by using a paired-comparison procedure to determine a spatial representation (Schneider, 1980a; Schneider, Parker, \& Stein, 1974). On any single trial, a subject is presented with two pairs of tones differing in frequency and intensity, and asked to indicate whether the tones $(x, y)$ in the first pair were more or less dissimilar than the tones $(w, z)$ in the second pair. Note that this requires only a binary judgment on the part of subjects and that no numerical estimates are involved. This technique assumes that tone pair $(x, y)$ will be judged more dissimilar than tone pair $(w, z)$ if and only if the distance, $D(x, y)$, between tones $x$ and $y$ is greater than the distance, $D(w, z)$, between tones $w$ and $z$. These binary comparisons can be used to locate the stimuli in a spatial array such that the in- terpoint distances among these stimuli best predict the pair-comparison data (Schneider, 1980b). Three subjects were extensively tested in the present study to permit the construction of individual spatial representations in order to see whether these representations differed from subject to subject and to determine which of the four perceptual attributes served as dimensions of this space.

\section{METHOD}

\section{Subjects}

One of the authors and two research assistants in the Department of Psychology, University of Toronto, served as subjects in this study. All were paid for their cooperation. None had any hearing defects, and all were experienced observers.

\section{Apparatus}

The subject presented himself with one of the two pairs of tones to be compared by pressing Button 1. Pressing Button 2 resulted in the presentation of the second pair. The tones in a pair were $750 \mathrm{msec}$ in duration and were separated by a silent period of $900 \mathrm{msec}$. Each of the tones in a pair was generated by a separate oscillator (Hewlett-Packard 204C). The output intensity of each oscillator was controlled by a Hewlett-Packard attenuator (Model 350D). When the subject pressed Button 1, the output of the first attenuator was gated to an amplifier by means of a GrasonStadler electronic switch (Model 1287B). Fifty milliseconds later, the electronic switch was turned on for a period of $750 \mathrm{msec}$, with a rise and decay time of $10 \mathrm{msec}$ to dampen transients. Following an 850 -msec silent period, the same sequence was repeated for Attenuator 2 . In both cases, the output of the electronic switch, after being amplified (Tektronix AM 502), drove the earphone presenting the tones to the subject. As far as the subject was concerned, pressing Button 1 resulted in the presentation of two 750msec tones, differing in frequency and intensity, separated by a silent period of $900 \mathrm{msec}$. If Button 2 was pressed, the same sequence of events occurred, except that the input to the electronic switch was supplied by two additional oscillator-attenuator pairs to generate a different pair of tones. The subject was allowed to present himself with either pair any number of times he wished in order to arrive at a judgment of which pair was more dissimilar. A 50 -msec delay occurred after the presentation of either pair before a subsequent buttonpress would produce another pair of tones.

Tones were presented to the right ear of the subject via Beyer Model DT48S headphones which were calibrated by the National Bureau of Standards in Washington, D.C., using an NBS 9-A coupler. Frequency values were set manually by Experimenter 1, while the intensity values were set by Experimenter 2. Prior to each experimental session, output intensities of the oscillators were calibrated using a Tektronix digital voltmeter (Model DC 501). Frequency was monitored throughout the session by a Tektronix frequency counter (Model DC 503). A Tektronix oscilloscope was used in conjunction with these instruments to insure that no waveform clipping or distortion was present.

The subject was seated in a single-walled IAC sound-resistant booth and communicated with the experimenter via an intercom.

\section{Procedure}

The subject was instructed to report which pair of tones had the greater subjective difference or dissimilarity. The frequency and intensity values of the 11 tones employed in this experiment are listed in Table 1 . With 11 tones, there are 55 distinct tone pairs and 1,485 distinct comparisons of pairs. During the course of 22 experimental sessions, a subject was presented with each of these 1,485 comparisons exactly once. The order of presentation of these tone pairs was completely randomized. An experimental session consisted of 70 comparisons with a short break after the first 35 trials. 
Table 1

Stimulus Parameters

\begin{tabular}{ccc}
$\begin{array}{c}\text { Stimulus } \\
\text { Number }\end{array}$ & $\begin{array}{c}\text { Frequency (in } \\
\text { Hertz) }\end{array}$ & $\begin{array}{c}\text { Intensity (in } \\
\text { Decibels SPL) }\end{array}$ \\
\hline 1 & 325 & 72 \\
2 & 625 & 80 \\
3 & 925 & 50 \\
4 & 1225 & 60 \\
5 & 1225 & 104 \\
6 & 1550 & 98 \\
7 & 1850 & 67 \\
8 & 2150 & 92 \\
9 & 2450 & 80 \\
10 & 2750 & 88 \\
11 & 3050 & 56 \\
\hline
\end{tabular}

Five replications were performed with each subject, giving a total of five judgments for each of the 1,485 possible comparisons. The order of presentation of tones within a pair was completely randomized across replications and subjects.

\section{RESULTS}

The data from this experiment consisted of the number of times each subject judged one pair of tones to be more dissimilar than another for each of the 1,485 comparisons. A geometrical representation for these dissimilarity comparisons would suggest that tone pair $(r, s)$ would be judged more dissimilar than tone pair $(t, u)$ if and only if the distance, $D(r, s)$, between tones $r$ and $s$ in the spatial representation was greater than the distance, $D(t, u)$, between tones $t$ and $u$ in the same representation. Since there were five replications and three subjects, each comparison was judged a total of 15 times. Whenever pair $(r, s)$ was judged to be more dissimilar than pair $(t, u) 8$ or more times out of 15 , we wrote $(r, s)>(t, u)$, and took this as implying that $D(r, s)>D(t, u)$.

A Euclidean distance function in two dimensions was assumed. In this model, $(r, s)>(t, u)$ implies $D(r, s)>D(t, u)$, where $D(r, s)$ and $D(t, u)$ are Euclidean distances in a two-dimensional space. A modified version (see Appendix) of a nonmetric program designed for paired comparisons of differences (Schneider, 1980b) was used to determine a twodimensional spatial configuration for the stimuli. The program searches for the two-dimensional configuration which best predicts the paired comparison data; that is, it tries to arrange the points in a twodimensional space such that whenever $(r, s)>(t, u)$, then $D(r, s)>D(t, u)$, where $D(r, s)$ is the Euclidean distance between points $r$ and $s$ in the spatial configuration. Of course, in errorful data it is not possible to do this perfectly; hence, the program minimizes the number of disagreements between the predicted paired comparisons and the obtained paired comparisons. Formally, the program minimizes the quantity $G=N_{d} /\left(N_{r}-N_{t}\right)$, where $N_{d}$ is the number of disagreements, $N_{r}$ is the number of paired com- parisons, and $N_{t}$ is the number of cases in which the predicted comparison is indeterminate, that is, $D(r, s)$ $=D(t, u)$. The value of $G$ for this two-dimensional solution was .083 .

The value of $G$ can be used to estimate the degree to which the distances in the recovered configuration actually reflect the "true" interpoint distances between the tones. To accomplish this, the index of metric determinacy, M, was estimated for the twodimesional solution. The index of metric determinacy (see Young, 1970) is the squared Pearson correlation coefficient between the true distances (those which presumably generated the paired comparisons) and the distances produced by the nonmetric scaling program. Hence, $M$ varies between 0 and 1 and $M=1$ means that the true distances have been perfectly reconstructed. In no empirical investigation using these techniques are the true distances known. However, it is possible to estimate $M$ from the number of points, number of dimensions and $\mathrm{G}$-all of which are available. The result is that if $M$ is sufficiently high (above .96 , say), it is reasonable to assume that the original configuration has been recovered up to a rotation, translation, or expansion of the axes. For the twodimensional configuration obtained in the present study, $M$ is estimated to be .97 . Hence, we can assume that we have constructed a reasonably good geometrical representation for the dissimilarity of pure tones.

Figure 1 shows the obtained two-dimensional spatial configuration. Since the orientation of the axes in a Euclidean space is arbitrary, the axes were rotated so as to maximize the correlation between the projections of the points onto the rotated axes and

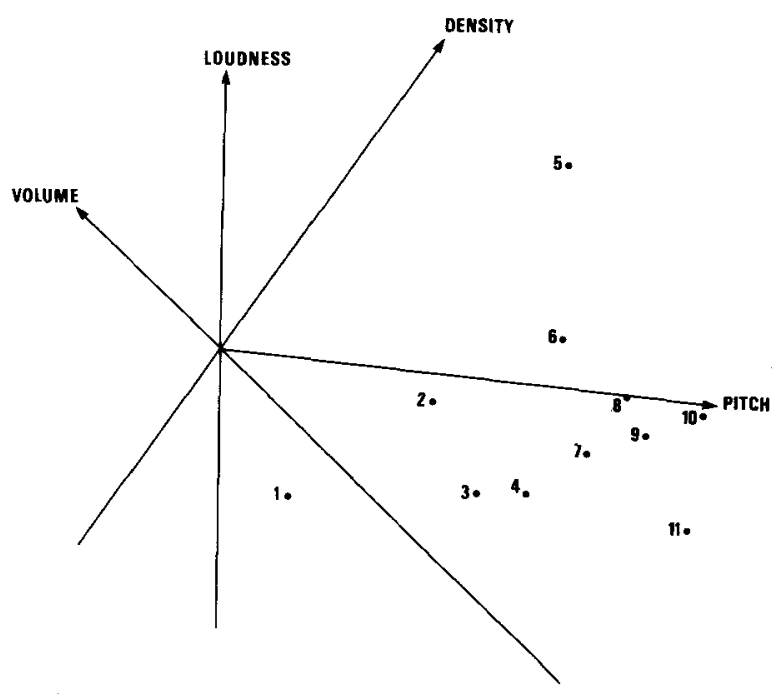

Figure 1. Two-dimensional Euclidean representation of the perceived differences among the tones in Table 1 for the combined data of three subjects. Best-fitting axes of loudness, pitch, volume, and density are shown. 
the loudness, pitch, volume, and density values of the tones. In finding the best rotation for loudness, we assumed a power function, since almost all investigators (see Marks, 1979, for a review) agree that loudness is a power function of sound intensity. Specifically, it was assumed that $X(r)=a L(r)+b=$ $a I(r)^{n}+b$, where $L(r)$ is the presumed loudness of tone $r, X(r)$ is its projection onto the rotated axis, and a, $\mathrm{n}$, and $\mathrm{b}$ are constants. Following Schneider et al. (1974), values of $a, n$, and $b$ were found which maximized the correlation coefficient between $\log [X(r)-$ b] and $n \log [I(r)]+\log$ a for successive .2-deg rotations of the axes. The rotated axis which maximized this correlation is labeled in Figure 1 as loudness, with the direction of the arrow indicating increasing loudness. The value of the exponent of the power function for loudness was .22 as a function of sound pressure.

The axes were also rotated to find the best-fitting axis to the perceived pitches of the tones. Three functions have been proposed to describe how pitch changes with frequency. The first is the musical scale which states that pitch is a logarithmic function of frequency. The second is the mel scale (Stevens \& Volkmann, 1940), which falls somewhere between a logarithmic scale and a linear scale of frequency over the range employed in this experiment. The third states that pitch may be described as a power function of frequency (Painton, Cullinan, \& Mencke, 1977). We have chosen the latter (the power function) as a description of the way in which pitch changes with frequency, since changes in the exponent in the range from 0 to 1.0 encompass the musical scale on one end (as the exponent approaches 0.0 , the function approaches a logarithmic form) and the linear scale on the other (an exponent of 1.0 defines a linear scale), with the mel scale in between (over this frequency range, intermediate exponents closely approximate the mel scale). By allowing the exponent to vary, we can determine, at least to a first approximation, which function better describes perceived pitch for these data. Thus, the axes were rotated until the best-fitting axis was found to the power-transformed frequencies. The same fitting procedure used for loudness was followed in this case. The axis displaced $96 \mathrm{deg}$ from the loudness axis provides the maximum correlation with a power function transformation of frequency (exponent $=.08$ ). Notice that the angle between the loudness dimension (the best fit to a power transformation of intensity) and the pitch dimension (the best fit to a power function of frequency) is close to 90 deg. This suggests that axes of pitch and loudness are orthogonal dimensions of the auditory space.

Although pitch and loudness appeared to give a good fit in two dimensions, we also rotated axes to obtain best correlations with volume and density. Accordingly, rotations were determined such that the projections onto an axis correlated best with volume in one case and with density in the other. Specifically, the axes were rotated until the correlation between $X(r)$ and $V(r)$ was maximized for volume and $X(r)$ and $D(r)$ was maximized for density. The volume values were computed from Terrace and Stevens (1962) according to the formula $V=k\left(I / I_{o}\right) g(F)$, where $V$ is volume, $I$ is the sound pressure of the tone, $I_{0}$ is the reference sound pressure of $20 \mathrm{~N} / \mathrm{m}^{2}, F$ is frequency, and $\mathrm{g}$ is a function relating the exponent of the power function for volume to frequency. The values of $g(F)$ were determined by reading off the values from the function relating exponent to frequency in Figure 3 from Terrace and Stevens (1962). The value of $k$ was derived assuming a convergence of the functions at $140 \mathrm{~dB}$ SPL. The density values were determined from the formula $D=c\left(I / I_{0}\right)^{h(F)}$, where $D$ is density, $\mathrm{c}$ is a constant, and $\mathrm{h}$ is the function relating the exponent of the power function for density to frequency. Values of $h(F)$ were read from Figure 7 in Guirao and Stevens (1964). The value of $c$ was computed assuming that the density functions converged at $155 \mathrm{~dB}$. The best axes for volume and density are also shown in Figure 1. Note that the volume and density axes are approximately orthogonal $(84 \mathrm{deg})$ to one another and that volume is displaced by an angle of $49 \mathrm{deg}$ from the loudness axis.

Figure 2 (upper left hand corner) plots the projections of the tones onto the best-fitting loudness dimension as a function of predicted loudness. The straight line represents perfect prediction. The upper right-hand corner shows the relation between pitch projections and predicted pitch, while the two bottom panels present the equivalent functions for volume and density. It is clear that all four axes provide reasonably good fits to the obtained configuration, with pitch exhibiting the best fit. With the exception of one point $(1,225 \mathrm{~Hz}, 104 \mathrm{~dB})$, the projections of the points onto the loudness axis are in reasonably good agreement with what would be predicted on the basis of a power function for loudness. Notice, however, that reasonably good fits are obtained for volume and density axes in the same configuration. A parsimonious interpretation of these data would therefore suggest that although the perceptual space for pure tones can be represented in only two dimensions, the two dimensions can be identified either as loudness and pitch or as volume and density, since loudness and pitch appear to be orthogonal to one another, as are volume and density (see Figure 1).

To determine the extent to which the group data characterized the individual subjects, separate analyses were carried out for the three subjects. For each subject, it was assumed that whenever $(r, s)$ was judged more dissimilar than $(t, u)$, three or more times out of five, $(r, s)>(t, u)$. Figures 3 and 4 show the spatial configurations obtained for E.D. and 


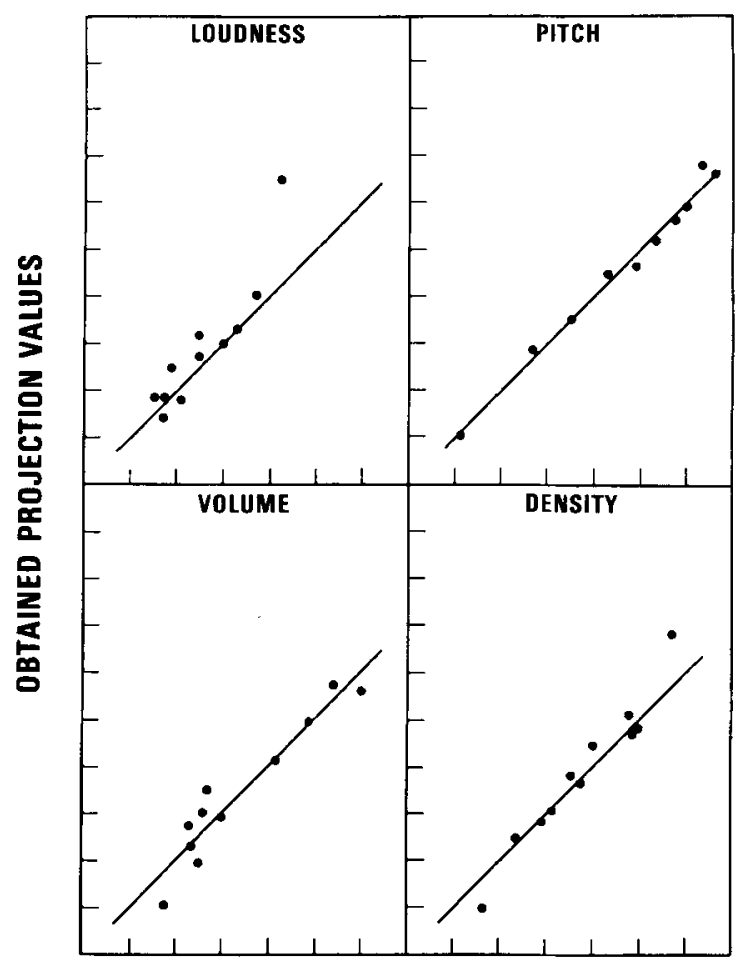

PREDICTED PROJECTION VALUES

Figure 2. Obtained projection values from the group data (see Figure 1) as a function of the predicted values for loudness, pitch, volume, and density.

F.M., respectively. Both subjects exhibited interpretable two-dimensional configurations. The values of G for E.D. and F.M. were .125 and .082 , respectively. These correspond to coefficients of metric determinacy of .96 and .97 . Thus, the configurations can be considered, for all practical purposes, as being unique up to rotation, expansion, and translation of axes. A comparison of these two configurations with the one obtained for the group data (Figure 1) shows that these two individual subjects are in good agreement with the group data. The best-fitting axes for loudness, pitch, volume, and density are also shown. For both of these subjects, the loudness and pitch axes are approximately orthogonal to one another (85 and $80 \mathrm{deg}$ for E.D. and F.M., respectively), as are the volume and density axes (102 and $100 \mathrm{deg}$ for E.D. and F.M.). Also, the fit of the data to the axes is reasonably good, as indicated in Figures 6 and 7. Figure 6 shows the relationship between the projections of the points onto the four perceptual axes (pitch, loudness, volume, and density) and their predicted values for E.D. Figure 7 presents the equivalent plots for Subject F.M. (The determination of predicted values was identical to that described for the group data.) Note that for both subjects the pitch axis provides a good fit to the configuration. How- ever, the value of the exponent for the pitch function is somewhat different for the two subjects (.24 and .33 for E.D. and F.M., respectively). The projections of E.D.'s configuration onto the loudness dimension are strongly related to predicted loudness (with an exponent as a function of sound pressure of .27), while F.M.'s projections are less systematically related to predicted loudness (with an exponent as a function of sound pressure of .30). As was the case for the group data, axes of volume and density also provided good fits to the configurations.

Figure 5 presents the two-dimensional configuration for Subject R.B. The value of $G$ for this configuration was .083 , while the coefficient of metric determinacy was .97 . Hence, we may consider this configuration as reasonably unique up to rotation, expansion, and translation. Note, however, that this configuration is clearly not as interpretable as those for the remaining two subjects. Of the four psychological attributes, the only one that can reasonably be

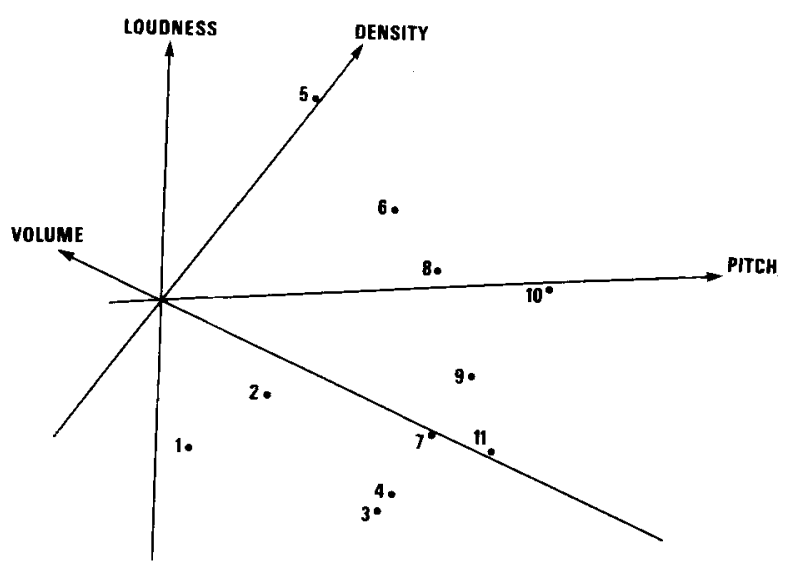

Figure 3. Two-dimensional Euclidean representation of the perceived differences among the tones in Table 1 for Subject E.D. Best-fitting axes of loudness, pitch, volume, and density are shown.

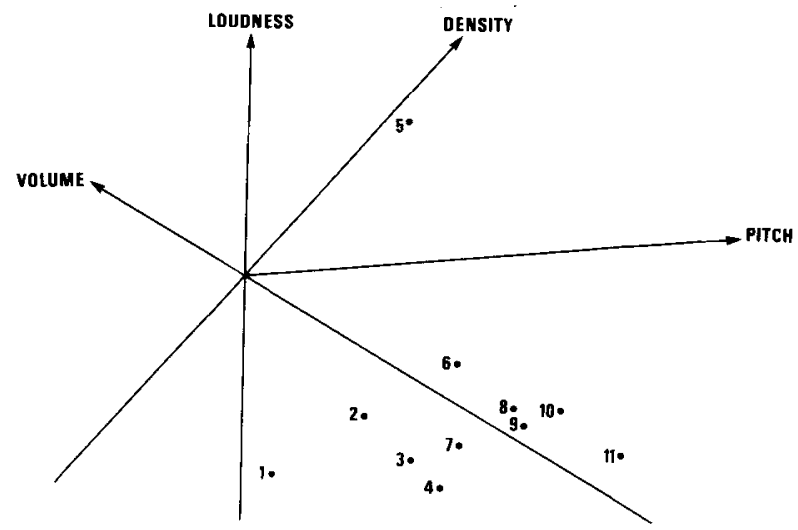

Figure 4. Two-dimensional Euclidean representation of the perceived differences among the tones in Table 1 for Subject F.M. Best-fitting axes of loudness, pitch, volume, and density are shown. 


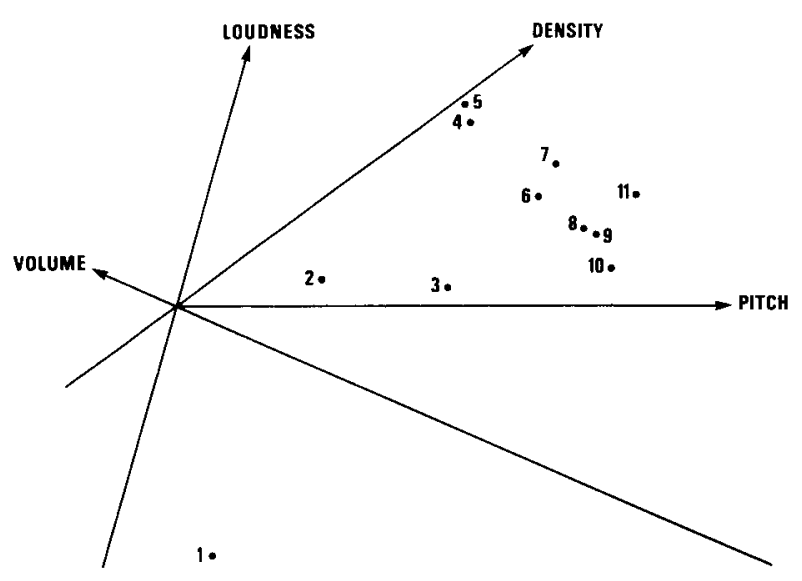

Figure 5. Two-dimensional Euclidean representation of the percelved differences among the tones in Table 1 for Subject R.B. Best-fitting axes of loudness, pitch, volume, and density are shown.

described as a dimension in this two-dimensional space is pitch where the fit between the predicted pitch values and the projections onto the pitch axis is good (see Figure 8). (The exponent for pitch for Subject R.B. is .02.) The relationship between the projections of the points onto the other perceptual axes and their predicted values is uniformly poor (see Figure 8). Furthermore, there is less of an indication that pitch and loudness or volume and density form orthogonal pairs of dimensions, since the loudness dimension is displaced only $73 \mathrm{deg}$ from the pitch dimension while volume is displaced $120 \mathrm{deg}$ from density (see Figure 5). Hence, it is unlikely that loudness, volume, and density are contributing to the comparisons of tone pairs. This is especially true for loudness because the relationship between predicted loudness and the loudness projection values is very poor.

The poor fit for three of the four dimensions suggested the possibility that R.B.'s judgments were based only on one dimension, namely, pitch. Accordingly, his data were analyzed to provide the best one-dimensional representation for the points. The value of $\mathrm{G}$ for this one-dimensional representation was .10. Note that this is only slightly higher than the value for his two-dimensional solution (.083). Thus, increasing the number of dimensions provided relatively little improvement in G. However, when onedimensional solutions were obtained for E.D. and F.M., G increased to .176 and .133 , respectively. Note that this is considerably higher than their twodimensional solutions (.125 and .082 , respectively). The relatively small improvement for R.B. in going from one to two dimensions suggests that his judgments of the dissimilarities of the tone pairs were based on a comparison of differences along a single psychological dimension. This, coupled with the good fit for the pitch dimension, suggests that R.B.'s comparisons of tone pairs were based solely on the perceived differences in pitches. The fact that his exponent for pitch was close to zero suggests that his pitch scale was approximately logarithmic with frequency, that is, the musical scale. On the other hand, the larger reduction in the value of $G$ for the other two subjects in going from one to two dimensions, coupled with the fact that interpretable dimensions were found for the two-dimensional configurations, shows that their judgments were based on differences in two psychological attributes-either pitch and loudness or volume and density.

\section{DISCUSSION}

For two of the subjects and for the group data, a two-dimensional tonal space is required in order to account for paired-comparison judgments of tonal dissimilarities. Figures 1, 3, and 4 show that bestfitting axes of loudness and pitch are approximately orthogonal to one another in these configurations. However, the same figures indicate that best-fitting axes of volume and density also exhibit orthogonality. Furthermore, the volume and density axes are approximately 45 -deg rotations of the loudness and pitch axes. An implication of this kind of representa-

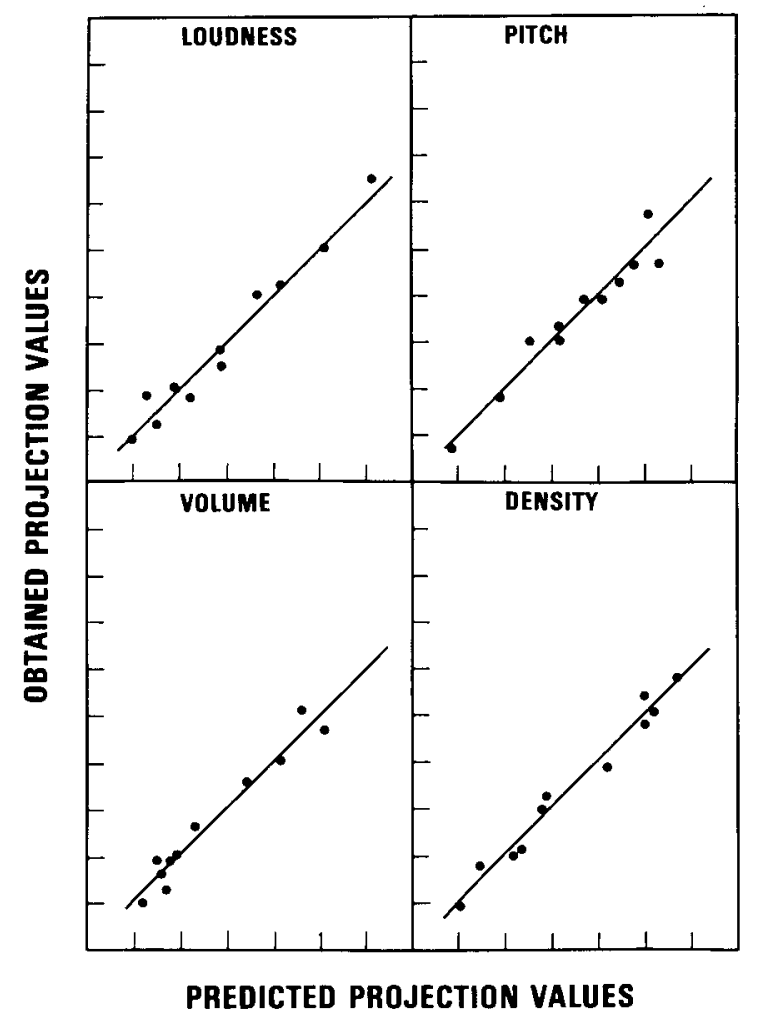

Figure 6. Obtained projection values from Subject E.D. (see Figure 3) as a function of the predicted values for loudness, pitch, volume, and density. 


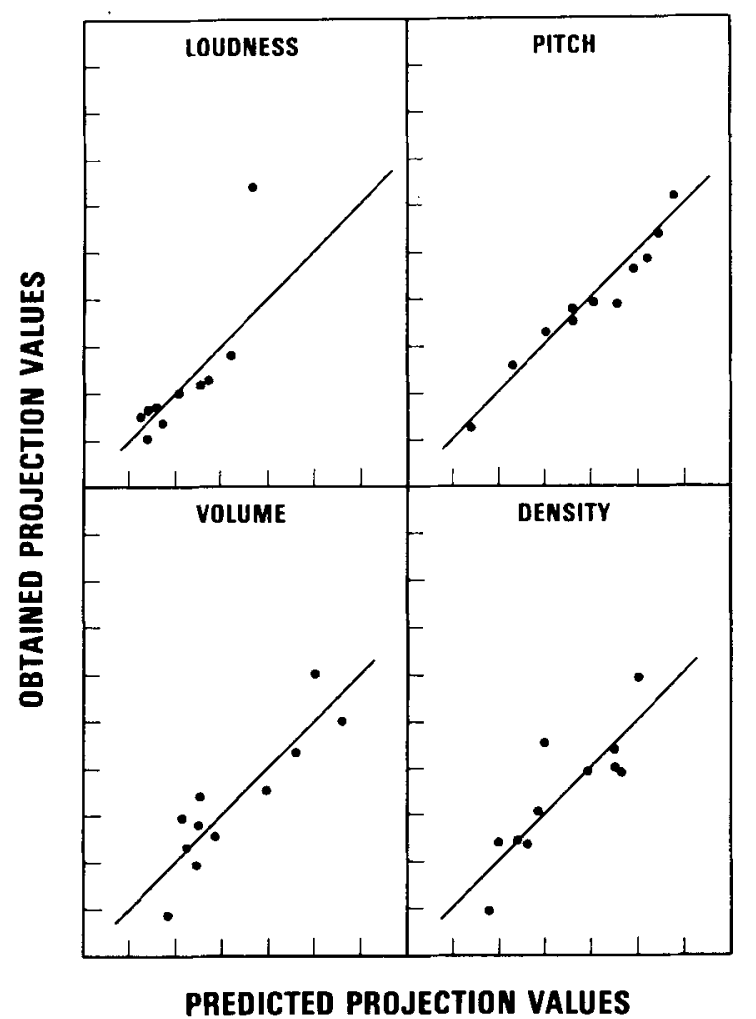

Figure 7. Obtained projection values from Subject F.M. (see Figure 4) as a function of the predicted values for loudness, pitch, volume, and density.

tion is that the volume or density of a tone is a simple function of its loudness and pitch values. If volume is displaced $45 \mathrm{deg}$ from loudness, then the volume of a tone is equal to a constant times the difference between the loudness and pitch values of the tone; that is, $V=c(L-P)$, where $L$ is loudness, $P$ is pitch, and $c=(1 / 2)^{1 / 2}$. Similarly, density is simply equal to a constant times the sum of loudness and pitch values of the tone; that is, $D=c(L+P)$. These two formulas follow directly from a 45-deg rotation of loudness and pitch axes.

In an attempt to see how well these formulas for volume and density can account for simple judgments of volume and density, we predicted the magnitude estimates of volume obtained by Terrace and Stevens (1962) and the magnitude estimates of density obtained by Guirao and Stevens (1964). In making these predictions, we assumed that $\mathrm{L}=121 \mathrm{I} .22-.19$, and $P=3.55 F .08-5.61$. These are exactly the values of the parameters estimated for the group solution in Figure 1. Hence, no additional parameter estimation was performed. Figure 9 presents the obtained magnitude estimates of volume (upper right panel) as a function of the predicted values based on our twodimensional model $[V=c(L-P)]$. Figure 9 (lower right panel) presents the equivalent data for density. Also shown in the left-hand panels are the obtained magnitude estimates as a function of predicted value for the Terrace and Stevens (1962) model for volume (upper left) and the Guirao and Stevens' (1964) model for density (lower left). Note that our present model predicts fairly accurately the magnitude estimates of volume and density from two different experiments. In fact, the correlation coefficients for the perceived vs. obtained functions for our model (.95 and .91 for volume and density, respectively) are almost as good as the correlation coefficients for the model developed to account for the original data (.99 and .96 for volume and density, respectively). Furthermore, parameter values for the Terrace and Stevens (1962) model and the Guirao and Stevens (1964) model were estimated so as to provide the best fit to the data. Our parameter estimates, on the other hand, were derived from a completely different experiment.

We also believe that our estimates of volume and density would have been more accurate had we been able to better specify the loudness of the tones. Several investigators (e.g., Schneider, Wright, Edelheit, Hock, \& Humphrey, 1972; Stevens, 1966) have shown that the exponent of the loudness function varies with frequency. In the present paper, we have made no attempt to estimate individual exponents for different frequencies. Thus, it is possible that cor-

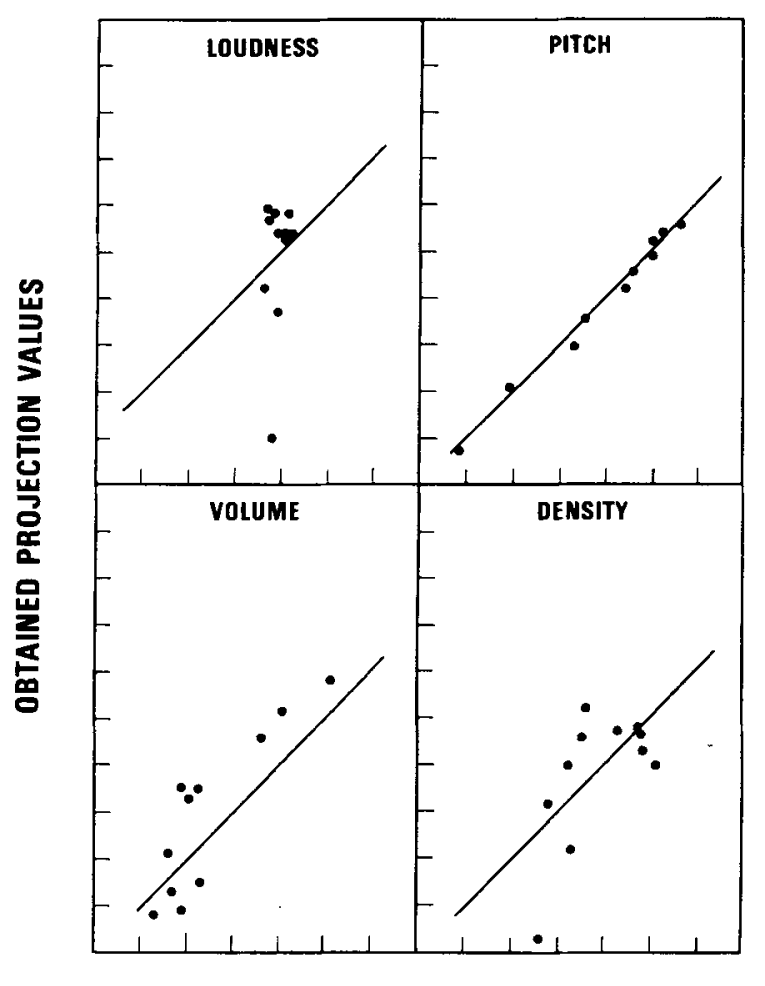

PREDICTED PROJECTION VALUES

Figure 8. Obtained projection values from Subject R.B. (see Figure 5) as a function of the predicted values for loudness, pitch, volume, and density. 


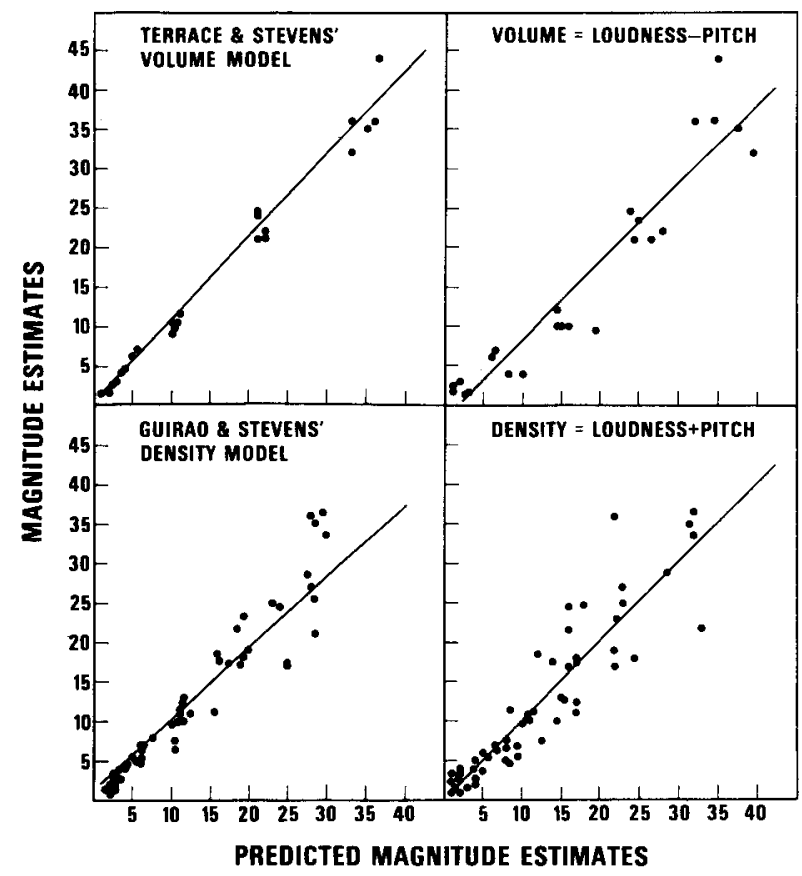

Figure 9. Upper left: Magnitude estimates of volume from Terrace and Stevens (1962) as a function of the predicted estimates from their model. Upper right: Magnitude estimates of volume from Terrace and Stevens (1962) as a function of predicted volume where predicted volume = loudness - pitch. Lower left: Magnitude estimates of density from Guirao and Stevens (1964) as a function of the predicted estimates from their model. Lower right: Magnitude estimates of density from Guirao and Stevens (1964) as a function of predicted density where predicted density $=$ loudness + pitch.

recting the loudness exponent for tonal frequency might improve the correlation between loudness projections and predicted loudness as well as improve the volume and density predictions. It should be noted that both of the models developed by Guirao and Stevens (1964) and Terrace and Stevens (1962) allow variation in exponent as a function of tonal frequency. A second factor that might conceivably have improved the fit of the model to the data would have been to correct for equal loudness. However, over the range of tones explored here, the equal-loudness contours are nearly flat (see Robinson \& Dadson, 1956), and when the intensity values were corrected for equal loudness, there was no significant improvement in fit.

Marks (1975), in attempting to describe the basis for visual-auditory synesthesia, proposed a twodimensional representation for auditory experience which is equivalent to the one found here. In Marks' two-dimensional representation, loudness and pitch were given as one set of orthogonal dimensions and volume and brightness (density) were identifed as a second orthogonal pair of dimensions displaced $45 \mathrm{deg}$ from loudness and pitch. Marks developed this description of auditory experience in an attempt to account for the interrelations between loudness, pitch, volume, and density, and to identify dimen- sions of auditory experience which correspond more or less to dimensions of visual experience. It is interesting that the same representation surfaces in an attempt to describe the perceived dissimilarity of tones.

The nonindependence of these four auditory dimensions has been noted earlier. Stevens et al. (1965) argued that magnitude estimates of loudness of narrowband noises were the product of their volume and density estimates; that is, $L=V \times D$. It should be noted that this formula is incompatible with the twodimensional representation proposed here because we would predict that loudness is the sum of volume and density $(\mathrm{L}=\mathrm{V}+\mathrm{D})$. Figure 10 replots the Stevens et al. (1965) data. The abscissa for the upper panel is the sum of the volume estimates and the density estimates. The abscissa for the lower panel is the product of the volume and density estimates divided by 20 . Figure 10 shows that the additive model predicts the obtained loudness estimates slightly better than the multiplicative model $(r=.97$ for the additive model and .96 for the multiplicative model). Hence, the geometrical model proposed here for the dimensions of auditory experience is consistent with the results of other studies of volume and density (Guirao \&

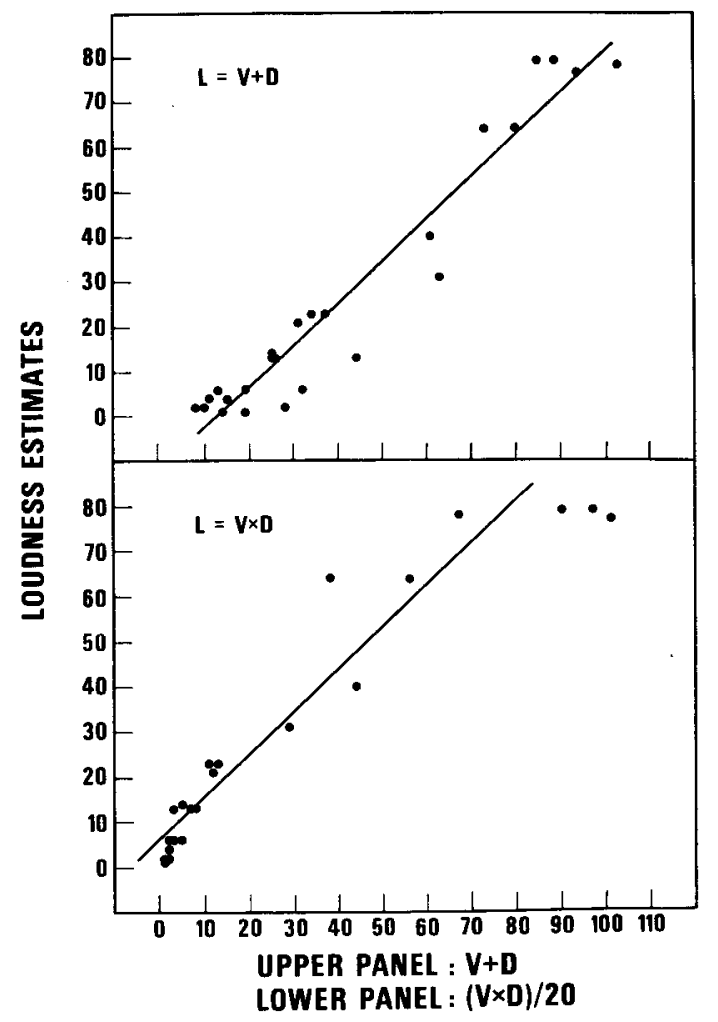

Figure 10. Upper panel: Magnitude estimates of the loudness of narrow-band noises from Stevens et al. (1965) as a function of the sum of the volume and density estimates for the same set of noises. Lower panel: Magnitude estimates of the loudness of narrowband noises from Stevens et al. (1965) as a function of the product of the volume and density estimates divided by 20 . 
\& Stevens 1964; Stevens et al., 1965; Terrace \& Stevens, 1962).

In proposing this model, we do not mean to imply that the manner in which the subject compares dissimilarities is by computing the Euclidean distance between the tones in a perceptual space. In fact, it is usually difficult to discriminate between the Euclidean metric and the so-called city-block metric (e.g., Behrman \& Brown, 1968; Carvellas \& Schneider, 1972). In the city-block metric, the distance between points in a space is the sum of their differences along the component dimensions. If the dimensions of the auditory space were loudness and pitch, a city-block metric would imply that the dissimilarity of two tones was the sum of their loudness difference and pitch difference. In many ways, this is a more appealing model psychologically, since it suggests a simpler cognitive algebra (see Anderson, 1977) for the combination of dissimilarities along perceptually analyzable attributes of a stimulus. We did not make any systematic attempt to determine the best city-block fits to the data because of difficulties in adapting the scaling routine to a city-block metric. However, our preliminary attempts confirmed what has long been known, namely, that it is difficult to discriminate between the two models on the basis of goodness of fit criteria since the two models yield spatial representations that are nearly identical.

Zagorski (1978) has sounded a further word of caution about inferring the nature of the combinatorial rule or cognitive algebra from dissimilarity judgments which appear to fit into a Euclidean space. Zagorski (1978) developed a plausible cognitive model for dissimilarity judgments for stimuli varying along more than a single psychological dimension which involved a weighted sum of the differences along the perceptually distinct dimensions. He was able to show that different values of the weighting factor can lead to distances that approximate those generated by Euclidean and city-block models. Thus, as Zagorski notes, data which fit a Euclidean model might, in fact, have been generated by a simpler cognitive process. The nature of this cognitive process certainly merits further study.

Further support for the cognitive nature of the judgments of tonal dissimilarity comes from a consideration of the one subject (R.B.) who apparently based his judgments primarily, if not exclusively, on the pitch differences among the stimuli. If the perceptual dissimilarity between two tones was an inflexible aspect of perceptual processing, we would not expect to find a subject who, in making his comparisons, did not take into account, or at least did not weight as heavily, dimensions of the stimuli other than pitch. This kind of processing flexibility might also explain why Chipman and Carey (1975) were unable to find orthogonal dimensions of pitch and loudness. Chipman and Carey (1975) averaged judgments across subjects in their study. If some subjects were basing their judgments only on a single dimension while others were judging along two dimensions, then it might not be surprising that orthogonal dimensions of pitch and loudness did not appear in their Euclidean solution. However, at the present time we do not have sufficient data on individual subjects to be able to assess how frequently such individual differences might occur and how drastically they might affect group results. Until we have this information, we should be cautious about inferring judgmental processes from group data.

It would be premature at this time to attempt to generalize these results to complex auditory stimuli such as musical tones and speech. While we might expect loudness and pitch to be dimensions of more complex stimuli, there is some reason to believe that if more complex stimuli are employed, other psychological dimensions may emerge and might possibly interact, in some unknown way, with these two more basic auditory dimensions. For example, identical musical notes played by different instruments have different overtone profiles which result in the perception of a difference in timbre. Consequently, we might expect judgments of dissimilarity to be based on loudness, pitch, and/or timbre. We might also expect additional dimensions to appear if comparisons were made among speech sounds, since speech sounds also vary over time. Hence, further research would be needed to determine the psychological dimensions of more complex auditory stimuli and the extent to which emergent dimensions in these cases interact or perhaps even overshadow the more basic attributes of pitch and loudness.

In the above discussion, we have implied that pitch and loudness should be considered as the dimensions of the tonal space, although equally good fits were found for volume and density. We have done this for three reasons. First, the dimensions of volume and density are not as compelling psychologically as those of pitch and loudness. In experiments on volume and density, the subjects are almost always carefully instructed as to the nature of these attributes. No such instruction is necessary for loudness and pitch. Second, the one subject who appeared to base his judgments on a single dimension used pitch rather than either volume or density. While this is admittedly weak support for the general argument, it tends to support the primacy of pitch and/or loudness as perceptual dimensions. Finally, if one considers the number of articles in the literature devoted to pitch and loudness as opposed to volume and density, it is clear that most perceptual psychologists consider volume and density as secondary aspects of the perceptual response to tones. Thus, if either pair of dimensions can account for the dissimilarity judg- 
ments, it seems more likely that the basis for tonal dissimilarity is likely to be the attributes of pitch and loudness.

\section{REFERENCES}

Anderson, N. H. Note on functional measurement and data analysis. Perception \& Psychophysics, 1977, 21, 201-205.

Behrman, B. W., \& Brown, D. R. Multidimensional scaling of form: A psychophysical analysis. Perception \& Psychophysics, $1968,4,19-25$.

Carvellas, T., \& Schneider, B. Direct estimation of multidimensional tonal dissimilarity. Journal of the Acoustical Society of America, 1972, 51, 1839-1848.

Chipman, S. F., \& Carey, S. Anatomy of a stimulus domain: The relation between multidimensional and unidimensional scaling of noise bands. Perception \& Psychophysics, 1975, 17, 417-424.

Guirao, M., \& Stevens, S. S. The measurement of auditory density. Journal of the Acoustical Society of America, 1964, 36, 1176-1182.

Marks, L. E. On colored-hearing synesthesia: Cross-modal translations of sensory dimensions. Psychological Bulletin, 1975, 82, 303-331.

Marks, L. E. A theory of loudness and loudness judgments. Psychological Review, 1979, 86, 256-285.

Painton, S. W., Cullinan, W. L., \& Mencke, E. O. Individual pitch functions and pitch-duration cross-dimensional matching. Perception \& Psychophysics, 1977, 21, 469-476.

Robinson, D. W., \& Dadson, R. S. A redetermination of the equal-loudness relations for pure tones. British Journal of Applied Physics, 1956, 7, 166-181.

SchNeIDER, B. Individual loudness functions determined from direct comparisons of loudness intervals. Perception \& Psychophysics, 1980, 27, 493-503. (a)

SCHNeIder, B. A technique for the nonmetric analysis of paired comparisons of psychological intervals. Psychometrika, 1980, 45, 357-372. (b)

Schneider, B., Parker, S., \& Stein, D. The measurement of loudness using direct comparisons of sensory intervals. Journal of Mathematical Psychology, 1974, 11, 259-273.

Schneider, B., Wright, A. A., Edelheit, W., Hock, P., \& HumphreY, C. Equal loudness contours derived from sensory magnitude judgments. Journal of the Acoustical Society of America, 1972, 51, 1951-1959.

Stevens, S. S. The attributes of tones. Proceedings of the National Academy of Sciences, 1934, 20, 457-459.

Stevens, S. S. Power-group transformations under glare, masking, and recruitment. Journal of the Acoustical Society of America, $1966,39,725-735$.

Stevens, S. S., Guirao, M., \& Slawson, A. W. Loudness: A product of volume times density. Journal of Experimental Psychology, 1965, 69, 503-510.

Stevens, S. S., \& Volkmann, J. The relation of pitch to frequency: A revised scale. American Journal of Psychology, 1940, 53, 329-353.

Terrace, H. S., \& Stevens, S. S. The quantification of tonal volume. American Journal of Psychology, 1962, 75, 596-604.

Young, F. W. Nonmetric multidimensional scaling: Recovery of metric information. Psychometrika, 1970, 35, 455-473.

ZAGorski, M. A. Noncombination of pitch and loudness in multidimensional scaling. Journal of the Acoustical Society of America, 1978, 63, 273-274.

\section{APPENDIX}

Schneider (1980b) developed an algorithm to search for the best one-dimensional representation for a set of stimuli whose psychological "distances" have been compared. This is equivalent to determining the location of each stimulus (its coordinate value) along a straight line segment. Initially, the stimuli are arbitrarily assigned locations (coordinate values) along the line segment. The program begins by taking the first stimulus and moving it alternately to the left and right along the line segment until either $G$ is reduced or the distance of the point from its original position on the line segment exceeds a criterial value. If $G$ is reduced, the stimulus is moved to the location that reduced $\mathrm{G}$. If $\mathrm{G}$ is not reduced before the boundaries are exceeded, then the stimulus remains in the last position at which $G$ was at its original value. Thus, the program attempts to move the stimulus to a location that reduces $G$. It then moves on to another stimulus along the line segment and repeats this process until there is no further improvement in $\mathrm{G}$.

The two-dimensional version of this program again begins with an artificial starting configuration, this time, however, in two dimensions. Starting with the first stimulus in the configuration, the program shifts the location of the stimulus in two dimensions in a systematic fashion to attempt to find a location for that stimulus which results in a reduction of $\mathbf{G}$, that is, a location which results in a better prediction of the paired comparison data. In the first phase of the program, each stimulus is moved in a steplike approximation to a spiral pattern; that is, each stimulus spirals out from its original location until it finds a position that reduces $\mathrm{G}$ or until it exceeds the boundaries for the configuration. This first phase is continued until the step size becomes so small that the time it takes to complete an iteration becomes prohibitive. At that point, further movement of the point (Phase 2) is with respect to changes in its $X$ coordinate value (holding the $Y$ value fixed) alternating with changes in the $Y$ coordinate value (holding the $X$ value fixed). Also, during both Phase 1 and Phase 2, after each complete iteration through the set of stimuli, the ordinate was systematically expanded and contracted (holding the abscissa fixed) in an attempt to find an expansion or contraction that reduced $\mathrm{G}$.

The ability of the program to recover a configuration was tested using the Monte-Carlo procedures described by Young (1970) and used by Schneider (1980b). Briefly, comparisons of distances were determined from a random configuration of points in two dimensions and used as input to the program. With no error term added to the coordinate values, the average value of $G$ for 11 point configurations was .003 , with an index of metric recovery of .997. Hence, the program was able to recover the original configurations with a good degree of accuracy. To obtain estimates of metric determinacy from values of G, progressively larger error terms were added to the coordinate values before computing the distances, following the procedure developed by Young (1970) and used by Schneider (1980b). The estimates of $M$ found in this paper were based on these simulations. To avoid local minima, different starting configurations were employed. The final solutions shown in Figures 1, 3, 4, and 5 represent the lowest values of $\mathrm{G}$ found from employing a number of different starting configurations.
(Manuscript received February 5, 1981; revision accepted for publication May 1, 1981.) 